\title{
E-Skin Pressure Sensors Made by Laser Engraved PDMS Molds ${ }^{+}$
}

\author{
Andreia dos Santos, Nuno Pinela, Pedro Alves, Rodrigo Santos, Elvira Fortunato, Rodrigo Martins, \\ Hugo Águas and Rui Igreja * \\ CENIMATIi3N, Departamento de Ciência dos Materiais, Faculdade de Ciências e Tecnologia, Universidade \\ Nova de Lisboa, Campus de Caparica, 2829-516 Caparica, Portugal; ass.santos@campus.fct.unl.pt (A.d.S.); \\ n.pinela@campus.fct.unl.pt (N.P.); p.u.alves@gmail.com (P.A.); rcd.santos@campus.fct.unl.pt (R.S.); \\ emf@fct.unl.pt (E.F.); rm@uninova.pt (R.M.); hma@fct.unl.pt (H.Á.) \\ * Correspondence: rni@fct.unl.pt; Tel.: +351-21-294-8562 \\ + Presented at the Eurosensors 2018 Conference, Graz, Austria, 9-12 September 2018.
}

Published: 12 November 2018

\begin{abstract}
This work describes the production of electronic-skin (e-skin) piezoresistive sensors, which micro-structuration is performed using laser engraved molds. With this fabrication approach, low-cost sensors are easily produced with a tailored performance. Sensors with micro-cones and a high sensitivity of $-1 \mathrm{kPa}^{-1}$ under $600 \mathrm{~Pa}$ are more adequate for the blood pressure wave detection, while sensors micro-structured with semi-spheres and a maximum sensitivity of $-6 \times 10^{-3} \mathrm{kPa}^{-1}$ in a large pressure range $(1.6 \mathrm{kPa}$ to $100 \mathrm{kPa})$ are more suitable for robotics and functional prosthesis.
\end{abstract}

Keywords: electronic skin; piezoresistivity; laser engraving; blood pressure; robotics; prosthesis

\section{Introduction}

Human skin is an inspiring organ due to its outstanding performance, namely in the perception of several mechanical stimuli [1]. Electronic skin (e-skin) is a flexible and stretchable electronic surrogate of skin with sensing properties [1] that shows an enormous potential for human health monitoring, humanoid robots, and functional prosthetic devices [2-5]. E-skin pressure sensors use some distinct effects for pressure transduction [6-8], but the attractive simplicity of piezoresistivity has fuelled a great research in the field [9-12]. When playing on contact resistance change, the microstructuration of sensors' films increases the contact area between them, hence increasing the RoFf/RoN ratio [12] and thus improving the sensitivity. Sensors typically have a higher sensitivity for low pressures and a significantly lower value for higher pressures [2,6,10-13]. For the blood pressure wave detection at the wrist, a sensor should have a high sensitivity below $400 \mathrm{~Pa}$ [9]. For robotics and prosthesis, a constant sensitivity in a range from less than $10 \mathrm{kPa}$ (gentle touch) to $100 \mathrm{kPa}$ (objects manipulation) is preferable [6]. Also, the majority of micro-structured sensors either use costly and time consuming photolithography techniques $[2,6,8,9,11,12]$ or low customizable techniques based on using objects as molds [10,13] for the micro-structuration. Hence, there is a need for a cheap and easy-to-produce pressure sensor with a high and constant sensitivity in a wide pressure range. Herein, an easy and fast production of highly customizable molds through laser engraving is explored, and the versatility of this technique is demonstrated through the production of piezoresistive e-skin sensors with different performances which makes them more suitable for health monitoring applications or robotics and functional prosthesis. 


\section{Materials and Methods}

The sensors were produced following a methodology described in a previous work [14]. The engraved patterns consisted in crosses or circles with a width/diameter of $100 \mu \mathrm{m}$ or $200 \mu \mathrm{m}$ and a pitch of $150 \mu \mathrm{m}, 200 \mu \mathrm{m}$, or $300 \mu \mathrm{m}$, over $4 \mathrm{~cm}^{2}$. The molds were either hard-PDMS (h-PDMS, 1:5 w/w ratio of curing agent to elastomer) for the engraving of circles or acrylic plates for the engraving of crosses. The laser speed was set to $0.1524 \mathrm{~m} \mathrm{~s}^{-1}$ (for acrylic) or $0.254 \mathrm{~m} \mathrm{~s}^{-1}$ (for h-PDMS), with a laser power from $2.5 \mathrm{~W}$ to $25 \mathrm{~W}$. Micro-structured standard-PDMS (s-PDMS, 1:10 w/w ratio) films were observed with a tabletop scanning electron microscope (SEM) (Hitachi TM3030Plus). Several weights were placed on the sensors to apply pressures from $15 \mathrm{~Pa}$ to $100 \mathrm{kPa}$, under $1 \mathrm{~V}$ to $10 \mathrm{~V}$. The relaxation time was estimated with the unloading of a small magnet (184 Pa). Semi-spheres sensors were mounted in a robotic arm, controlled by a servomotor, to monitor the pressure exerted during the grasping of an object, which was performed 2500 times. Sensors with cones were tested at the wrist for the monitor of the blood pressure pulse wave.

\section{Results and Discussion}

\section{1. h-PDMS Patterning}

The effect of laser power on h-PDMS molds was studied by varying the power from $25 \mathrm{~W}$ to $12.5 \mathrm{~W}, 7.5 \mathrm{~W}$, and $2.5 \mathrm{~W}$, while fixing the laser speed at $0.254 \mathrm{~m} / \mathrm{s}$ for a design based on circles with a pitch of $200 \mu \mathrm{m}$. Figure 1 shows that the height of the s-PDMS micro-structures peeled off from such molds increases with the laser power and also with the diameter, because while the laser is engraving a larger area, it melts more h-PDMS in depth. To achieve a semi-sphere like microstructure, a laser power of $7.5 \mathrm{~W}$ and a designed diameter of $200 \mu \mathrm{m}$ should be used because the resultant micro-structures are rounded and their ratio of height/diameter is the closest to 0.5 .
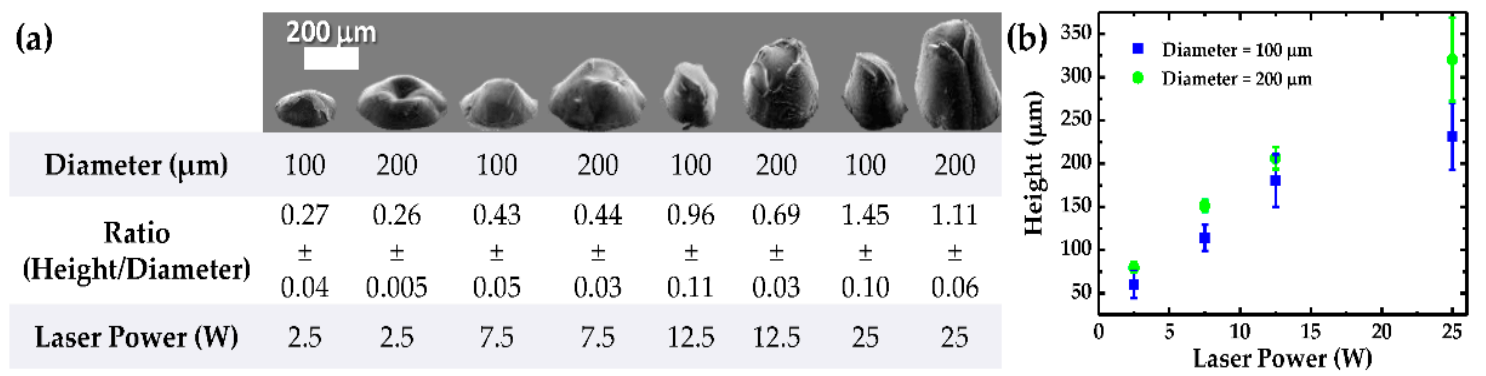

Figure 1. Effect of laser power on micro-structures peeled off from h-PDMS molds. (a) SEM images of micro-structures with the respective height/diameter ratio. (b) Height variation with laser power.

\subsection{Characterization of Micro-Structured Films Peeled off from h-PDMS Molds}

Figure 2a-d show a general view of s-PDMS films with semi-spheres, where the homogeneity of the micro-structures and their correct alignment is evident. Figure $2 \mathrm{e}-\mathrm{h}$ prove that PMMA and carbon coating seem to spread homogeneously over the semi-spheres surface. Additionally, the PMMA layer is thin enough to the point of having a null impact in the features of the semi-spheres (height, diameter, and pitch), as shown in the bottom of Figure 2. 


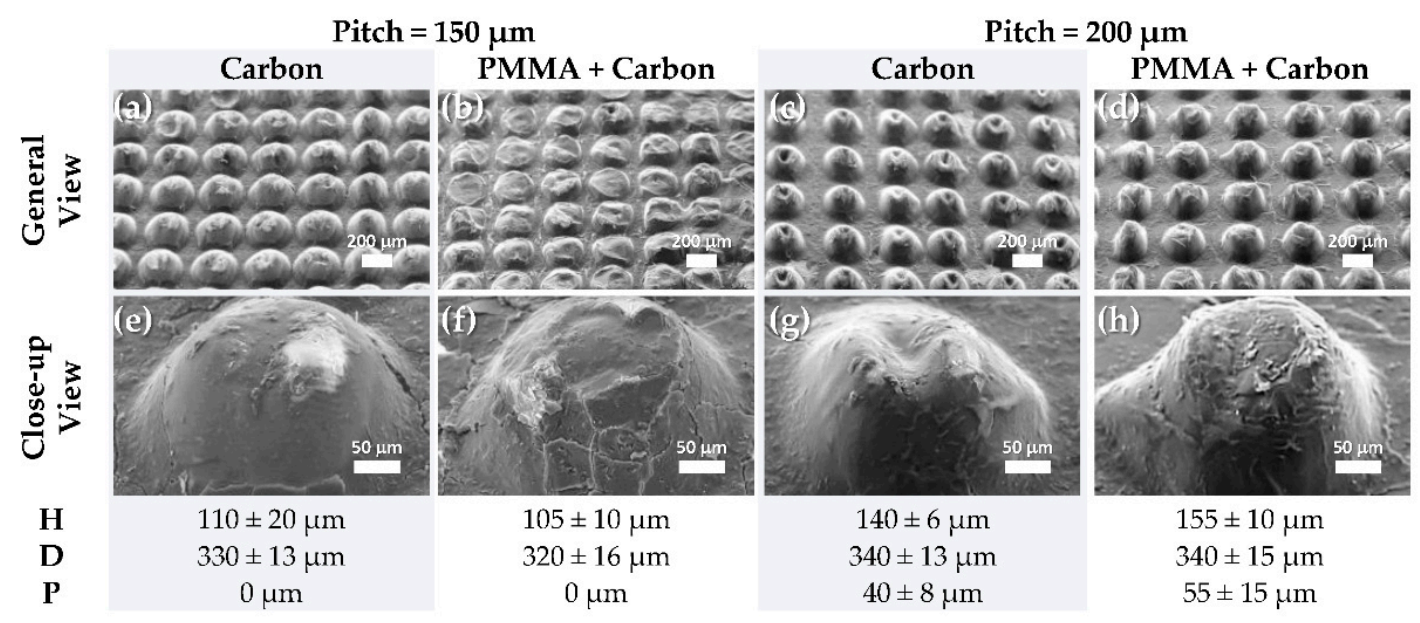

Figure 2. SEM images of s-PDMS semi-spheres (diameter $=200 \mu \mathrm{m})$. (a-d) General view of semispheres with a theoretical pitch of $150 \mu \mathrm{m}$ (a and b) or $200 \mu \mathrm{m}(\mathbf{c}, \mathbf{d})$ and carbon coating only (a,c) or both PMMA and carbon coating $(\mathbf{b}, \mathbf{d})$. (e-h) Close-up view of semi-spheres with a theoretical pitch of $150 \mu \mathrm{m}(\mathbf{e}, \mathbf{f})$ or $200 \mu \mathrm{m}(\mathbf{g}, \mathbf{h})$ and carbon coating only (e,g) or both PMMA and carbon coating (f,h). At the bottom are the average values \pm standard deviation of the real height $(\mathrm{H})$, diameter $(\mathrm{D})$, and pitch (P) of the semi-spheres.

\subsection{Electrical Characterization of Sensors}

A schematic illustration and a photograph of a semi-spheres sensor are shown in Figure 3a-b, respectively. These sensors may be subjected to bending and compressive forces without an impairment of their performance. Figure 3d illustrates the relative resistance change for cones sensors (produced with acrylic molds engraved with crosses) and semi-spheres sensors (produced with hPDMS molds engraved with circles with the optimized parameters from section 3.1) with different pitches, and the respective sensitivities (calculated as done elsewhere $[12,13]$ ) are presented in Figure 3e. Cones sensors generally have higher sensitivities for lower pressures, with a maximum of $-1 \mathrm{kPa}^{-1}$ under $600 \mathrm{~Pa}$ for a pitch of $300 \mu \mathrm{m}$. However, semi-spheres sensors were tested until higher pressures and revealed a maximum sensitivity of $-6 \times 10^{-3} \mathrm{kPa}^{-1}$ from $1.6 \mathrm{kPa}$ to $100 \mathrm{kPa}$ when having a pitch of $200 \mu \mathrm{m}$.

The relaxation time for semi-spheres sensors was estimated as shown in Figure $3 \mathrm{f}$, and the impact of the PMMA layer on this parameter was also evaluated. The PMMA layer seems not only to reduce the relaxation time of the sensors but also to smooth the sensors' behavior during relaxation. Contrarily, sensors without PMMA have a peak before stabilizing their resistance.

To study the reproducibility and effectiveness of the sensors, semi-spheres sensors were subjected to a cyclic compression (2500 cycles) done with a robotic arm upon grabbing an object (the setup of the test is shown in Figure $3 \mathrm{c}$ and the results are in Figure $3 \mathrm{~g}$ ) while cones sensors were placed at the wrist to monitor the blood pressure wave (Figure 3h). The semi-spheres sensor presents a reproducible response, with a similar relative resistance change for the first and last cycles of $(-33 \pm 1) \%$ and $(-32 \pm 1) \%$, respectively. The relative current change of the cones sensor placed at the wrist is coincident with the shape of the blood pressure wave. 
(a)

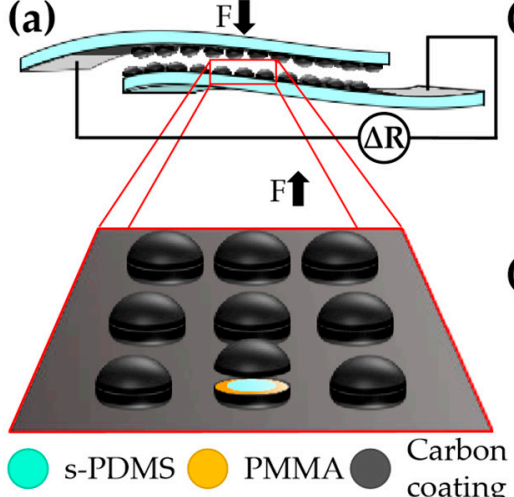

(e)

\begin{tabular}{|c|c|c|c|c|c|}
\hline \multirow[t]{2}{*}{ (e) } & \multirow[b]{2}{*}{$\begin{array}{l}\text { Pitch } \\
(\mu \mathrm{m})\end{array}$} & \multicolumn{2}{|c|}{ Cones } & \multicolumn{2}{|c|}{$\begin{array}{l}\text { Semi- } \\
\text { spheres }\end{array}$} \\
\hline & & 150 & 300 & 150 & 200 \\
\hline \multirow{2}{*}{$\begin{array}{l}0 \\
0 \\
0 \\
0 \\
0 \\
0 \\
3 \\
0 \\
\end{array}$} & $\begin{array}{c}\mathrm{S} \\
\left(\mathrm{kPa}^{-1}\right)\end{array}$ & -0.9 & -1 & -1 & -0.2 \\
\hline & $\begin{array}{c}\text { Range } \\
\text { (kPa) }\end{array}$ & $\begin{array}{c}0 \\
- \\
0.3\end{array}$ & $\begin{array}{c}0 \\
- \\
0.6\end{array}$ & $\begin{array}{c}0 \\
- \\
0.2\end{array}$ & $\begin{array}{c}0 \\
- \\
0.6\end{array}$ \\
\hline \multirow{2}{*}{ 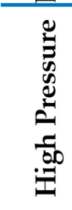 } & $\begin{array}{c}\mathrm{S} \\
\left(\times 10^{-3}\right. \\
\left.\mathrm{kPa}^{-1}\right)\end{array}$ & -8 & -10 & -2 & -6 \\
\hline & $\begin{array}{c}\text { Range } \\
\text { (kPa) }\end{array}$ & $\begin{array}{l}2.0 \\
- \\
10\end{array}$ & $\begin{array}{c}2.0 \\
- \\
10\end{array}$ & $\begin{array}{c}1.6 \\
- \\
100\end{array}$ & $\begin{array}{c}1.6 \\
- \\
100\end{array}$ \\
\hline
\end{tabular}
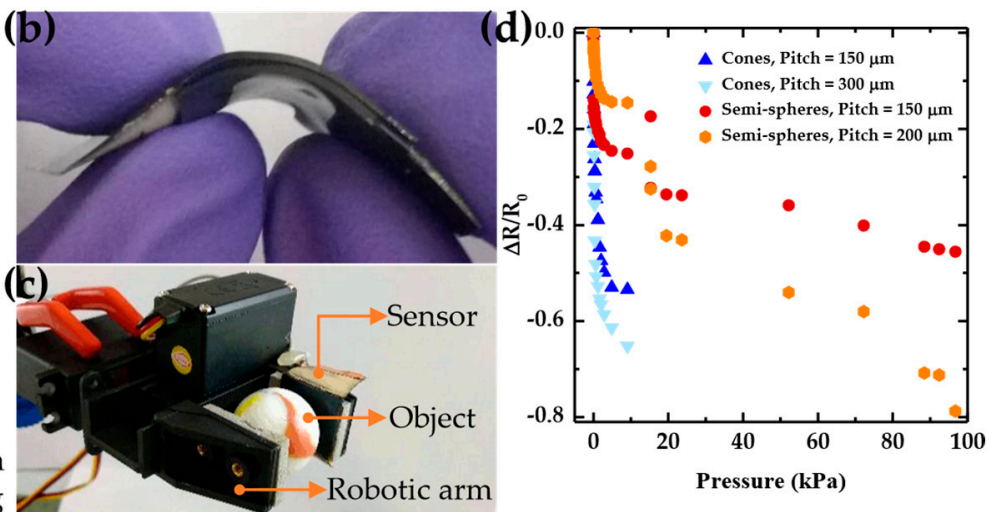

(f)

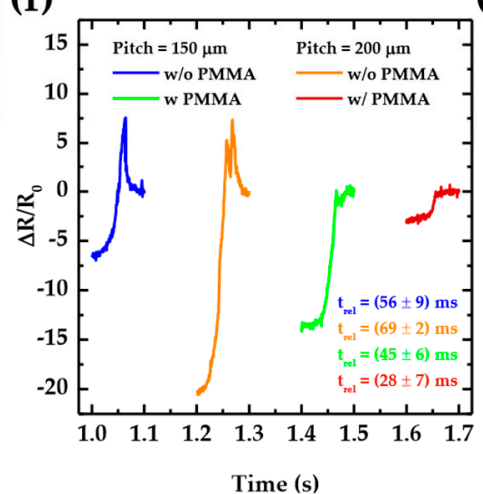

(g)

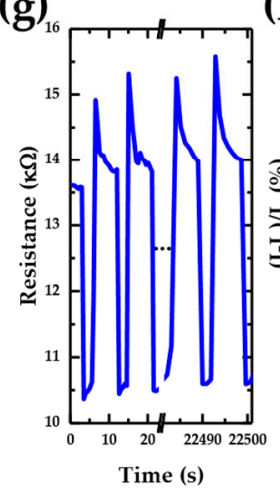

(h)

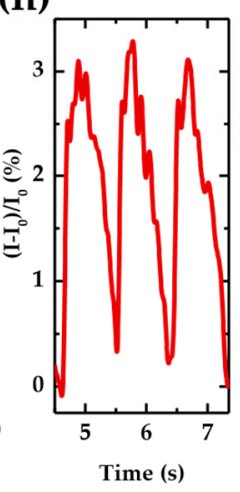

Figure 3. Electrical characterization of e-skin sensors. (a) Illustration of a semi-spheres sensor. (b) Photograph of a sensor under bending. (c) Photograph of a semi-spheres sensor placed in a robotic arm. (d) Relative resistance change for each sensor. (e) Sensitivity of the sensors from (d) for the low and high pressure regions. (f) Relaxation time of micro-domes sensors with (w) and without PMMA (w/o). The values indicated correspond to average values \pm standard deviation of 7 measurements. (g) Resistance change throughout time during the cyclic grasping and releasing of an object by a robotic arm with a semi-spheres sensor. (h) Relative current change throughout time during the detection of the radial artery blood pressure wave (at the wrist) with a micro-cones sensor.

Acknowledgments: The authors would like to acknowledge funding by FEDER funds through the COMPETE 2020 Programme and National Funds through FCT - Portuguese Foundation for Science and Technology under the project number POCI-01-0145-FEDER-007688, Reference UID/CTM/50025/2013 NOVA.ID.FCT. A. dos Santos acknowledges the support from the Portuguese Foundation for Science and Technology and MIT Portugal through the scholarship PD/BD/105876/2014.

Conflicts of Interest: The authors declare no conflict of interest. The founding sponsors had no role in the design of the study; in the collection, analyses, or interpretation of data; in the writing of the manuscript, and in the decision to publish the results.

\section{References}

1. Hammock, M.L.; Chortos, A.; Tee, B.C.K.; Tok, J.B.H.; Bao, Z. 25th anniversary article: The evolution of electronic skin (E-Skin): A brief history, design considerations, and recent progress. Adv. Mater. 2013, 25, 5997-6038, doi:10.1002/adma.201302240.

2. Schwartz, G.; Tee, B.C.-K.; Mei, J.; Appleton, A.L.; Kim, D.H.; Wang, H.; Bao, Z. Flexible polymer transistors with high pressure sensitivity for application in electronic skin and health monitoring. Nat. Commun. 2013, 4, 1859, doi:10.1038/ncomms2832.

3. Someya, T.; Kato, Y.; Sekitani, T.; Iba, S.; Noguchi, Y.; Murase, Y.; Kawaguchi, H.; Sakurai, T. Conformable, flexible, large-area networks of pressure and thermal sensors with organic transistor active matrixes. Proc. Natl. Acad. Sci. USA 2005, 102, 12321-12325, doi:10.1073/pnas.0502392102.

4. Gerratt, A.P.; Michaud, H.O.; Lacour, S.P. Elastomeric Electronic Skin for Prosthetic Tactile Sensation. Adv. Funct. Mater. 2015, 25, 2287-2295, doi:10.1002/adfm.201404365. 
5. Rocha, R.P.; Lopes, P.A.; De Almeida, A.T.; Tavakoli, M.; Majudu, C. Fabrication and characterization of bending and pressure sensors for a soft prosthetic hand. J. Micromech. Microeng. 2018, 28, 34001, doi:10.1088/1361-6439/aaa1d8.

6. Mannsfeld, S.C.B.; Tee, B.C.-K.; Stoltenberg, R.M.; Chen, C.V.H.-H.; Barman, S.; Muir, B.V.O.; Sokolov, A.N.; Reese, C.; Bao, Z. Highly sensitive flexible pressure sensors with microstructured rubber dielectric layers. Nat. Mater. 2010, 9, 859-864, doi:10.1038/nmat2834.

7. Dagdeviren, C.; Su, Y.; Joe, P.; Yona, R.; Liu, Y.; Kim, Y.-S.; Huang, Y.Y.; Damadoran, A.R.; Xia, J.; Martin, L.W.; et al. Conformable amplified lead zirconate titanate sensors with enhanced piezoelectric response for cutaneous pressure monitoring. Nat. Commun. 2014, 5, 4496, doi:10.1038/ncomms5496.

8. Fan, F.-R.; Lin, L.; Zhu, G.; Wu, W.; Zhang, R.; Wang, Z.L. Transparent Triboelectric Nanogenerators and Self-Powered Pressure Sensors based on Micropatterned Plastic Films. Nano Lett. 2012, 12, 3109-3114, doi:10.1021/nl300988z.

9. Pang, C.; Lee, G.-Y.; Kim, T.; Kim, S.M.; Kim, H.N.; Ahn, S.-H.; Suh, K.-Y. A flexible and highly sensitive strain-gauge sensor using reversible interlocking of nanofibres. Nat. Mater. 2012, 11, 795-801, doi:10.1038/nmat3380.

10. Wang, X.; Gu, Y.; Xiong, Z.; Cui, Z.; Zhang, T. Silk-molded flexible, ultrasensitive, and highly stable electronic skin for monitoring human physiological signals. Adv. Mater. 2014, 26, 1336-1342, doi:10.1002/adma.201304248.

11. Choong, C.-L.; Shim, M.-B.; Lee, B.-S.; Jeon, S.; Ko, D.-S.; Kang, T.-H.; Bae, J.; Lee, S.H.; Byun, K.-E.; Im, J.; et al. Highly stretchable resistive pressure sensors using a conductive elastomeric composite on a micropyramid array. Adv. Mater. 2014, 26, 3451-3458, doi:10.1002/adma.201305182.

12. Park, J.; Lee, Y.; Hong, J.; Ha, M.; Do Jung, Y.; Lim, H.; Kim, S.Y.; Ko, H. Giant Tunneling Piezoresistance of Composite Elastomers with Interlocked Microdome Arrays for Ultrasensitive and Multimodal Electronic Skins. ACS Nano 2014, 8, 4689-4697, doi:10.1021/nn500441k.

13. Zhu, Y.; Li, J.; Cai, H.; Wu, Y.; Ding, H.; Pan, N.; Wang, X. Highly sensitive and skin-like pressure sensor based on asymmetric double-layered structures of reduced graphite oxide. Sens. Actuators B Chem. 2018, 255, 1262-1267, doi:10.1016/j.snb.2017.08.116.

14. dos Santos, A.; Pinela, N.; Alves, P.; Santos, R.; Fortunato, E.; Martins, R.; Águas, H.; Igreja, R. Piezoresistive E-Skin Sensors Produced with Laser Engraved Molds. Adv. Electron. Mater. 2018, 4, 1800182, doi:10.1002/aelm.201800182.

(C) 2018 by the authors; Licensee MDPI, Basel, Switzerland. This article is an open access article distributed under the terms and conditions of the Creative Commons Attribution (CC BY) license (http://creativecommons.org/licenses/by/4.0/). 